This document is confidential and is proprietary to the American Chemical Society and its authors. Do not copy or disclose without written permission. If you have received this item in error, notify the sender and delete all copies.

\title{
DNA-mediated self-organization of polymeric nano- compartments leads to interconnected artificial organelles
}

\begin{tabular}{|r|l|}
\hline Journal: & Nano Letters \\
\hline Manuscript ID & nl-2016-03430b.R1 \\
\hline Danuscript Type: & Communication \\
\hline Complete List of Authors: & $\begin{array}{l}\text { Liu, Juan; University of Basel } \\
\text { Postupalenko, Viktoriia; University of Basel, Department of Chemistry } \\
\text { Loercher, Samuel; Universitat Basel } \\
\text { Wu, Dalin; Universitat Basel } \\
\text { Chami, Mohamed; University of Basel, Biozentrum } \\
\text { Meier, Wolfgang; University of Basel, Department of Chemistry } \\
\text { Palivan, Cornelia; University of Basel, Chemistry Department }\end{array}$ \\
\hline
\end{tabular}

\section{SCHOLARONE Manuscripts}




\title{
DNA-mediated self-organization of polymeric nano-
}

\section{compartments leads to interconnected artificial}

\section{organelles}

\author{
Juan Liu ${ }^{\dagger}$, Viktoriia Postupalenko ${ }^{\dagger}$, Samuel Lörcher ${ }^{\dagger}$, Dalin Wu ${ }^{\dagger}$, Mohamed Chami ${ }^{\dagger}$,Wolfgang \\ Meier $^{\dagger}$, Cornelia G. Palivan ${ }^{*}$, \\ †Department of Chemistry, University of Basel, Klingelbergstrasse 80, Basel 4056, Switzerland \\ "BioEM lab, Biozentrum, University of Basel, Mattenstrasse 26, 4058 Basel, Switzerland
}

\begin{abstract}
Self-organization of nano-components was mainly focused on solid nanoparticles, quantum dots or liposomes to generate complex architectures with specific properties, but intrinsically limited or not developed enough, to mimic sophisticated structures with biological functions in cells. Here, we present a biomimetic strategy to self-organize synthetic nanocompartments (polymersomes) into clusters with controlled properties and topology by exploiting DNA hybridization to interconnect polymersomes. Molecular- and external factors affecting the self-organization served to design clusters mimicking the connection of natural organelles: fine tune of the distance between tethered polymersomes, different topologies, no fusion of clustered polymersomes and no aggregation. Unexpected, extended DNA bridges that result from migration of the DNA strands inside the thick polymer membrane (about $12 \mathrm{~nm}$ ) represent a key stability and control factor, not yet exploited for other synthetic nano-object
\end{abstract}


networks. The replacement of the empty polymersomes with artificial organelles, already reported for single polymersome architecture, will provide an excellent platform for the development of artificial systems mimicking natural organelles or cells, and represents a fundamental step in the engineering of molecular factories.

KEYWORDS. self-organization, polymersome clusters, DNA functionalization, DNA migration, membrane contact sites

Self-organization is a process by which several components become ordered in space and/or time according to interaction rules, and generally characterized by emergent properties that differ from those of the single components. Almost all sophisticated biological functions and features of cells are realized by self-organization. ${ }^{1}$ The organization of the position and the connection between organelles determines their functions: for example, the spatial relationship between mammalian Golgi apparatus and the centrosomes changes during interphase and mitosis to achieve distinct signal pathways and functional interactions. ${ }^{2}$ In addition, the connection of specific organelles by membrane contact sites (MCSs) plays a central role in signal transduction, ${ }^{3,4} \mathrm{Ca}^{2+}$ storage, ${ }^{5,6}$ monogenesis, ${ }^{7}$ and act as a widespread mechanism operating in the cell's physiology and pathology. ${ }^{7-12}$ A biomimetic approach to self-organize synthetic compartments in order to achieve networks/clusters with a controlled spatial topology as in MCS connected organelles is of huge scientific and technological importance to model sophisticated 
biological functions, and to mimic biological systems to create intelligent and "living" materials or technological devices with application for example in medicine or catalysis.

Various solid nanoparticles (or nanorods), comprising mostly of inorganic materials have been organized into well-defined super-structures with emergent distinct and collective properties, ${ }^{13,14}$ such as fine-tunable optical, ${ }^{15,16}$ magnetic $^{17,18}$ and electrical ${ }^{19,20}$ responses in comparison with those of the single nano-objects. Among all molecular moieties used for self-organization of nano-objects, DNA is considered as one of the most powerful tools that favors highly regulated and complex structures including superlattices, ${ }^{21-24}$ colloidal molecules, ${ }^{25,} 26$ asymmetric nanoclusters $^{27,28}$ and chiral nanostructures. ${ }^{15,} 29$ The advantages of DNA arise from its remarkable inherent molecular recognition, feasible structural design by software, and rigid structure when hybridization takes place. ${ }^{30-32}$ In addition, the self-assembly of small nanoparticles into larger structures has been reported to improve their in vivo tumor accumulation, and facilitates their elimination after enzymatic degradation of DNA linkage. ${ }^{33}$ Despite the aforementioned advantages, the self-organization of solid nanoparticles is only rarely exploited for biomimetic architectures due to the lack of an aqueous core and potential cytotoxicity. ${ }^{34}$ Instead, nano-compartments comprising of liposomes or polymersomes are more appealing for biological applications and cell mimics upon self-organization because they can exhibit versatile functions by insertion of synthetic or biological molecules into their membrane and accommodating various active entities in their cavities. ${ }^{35-37}$ In this respect, we and others used polymersomes to design mimics of organelles by co-encapsulation of enzymes in tandem that were able to perform their activity inside the cavity of single polymersomes. ${ }^{36,38} \mathrm{Up}$ to now the design of artificial organelles has been focused on increasing the in situ complexity of the enzymatic reactions ${ }^{35}$ or the inner morphology by polymersome-in-polymersome architectures. ${ }^{39}$ 
In addition, polymersomes have the advantages of a variety of properties (wall thickness, polarity, non-toxicity or sensor-responsivity $)^{35}$ achieved by the chemical versatility of the polymer blocks and an improved mechanic stability compared to their counterpart, the liposomes (which contains intrinsic defects and can, depending on their composition, undergo membrane fusion). ${ }^{40-42}$ The increased stability of polymersomes is due to their significantly thicker membrane $(6-20 \mathrm{~nm})$ compared to the one of liposomes $(3-5 \mathrm{~nm})$, which results from the huge difference in the molecular mass between amphiphilic copolymers and lipids serving as building blocks for the nano-compartments. Diverse micrometer-size organized structures based on compartments have been realized by Pickering emulsion ${ }^{43-45}$ and microfluidics, ${ }^{46}$ where the biphasic system has to be exploited to stabilize the assembled structures. This limitation can be overcome by linking the compartments in aqueous solution through molecular moieties such as biotin-streptavidin ${ }^{47}$ and DNA, ${ }^{48-50}$ but the self-assembly process is poorly controlled, and leads in various cases to the formation of large aggregates. ${ }^{48,51}$ Templates are required in order to control the geometry of such micrometer-size assembled structures. ${ }^{52}$

Here, we present a strategy for self-organization of synthetic nano-compartments with controlled spatial topology based on the hybridization of complementary DNA strands exposed at the surface of the compartments. In addition, as our aim is to take advantage of the intelligence of nature in respect to organelles connected by MCSs, the self-organization of nanocompartments has to fulfill various bio-related requirements: (i) a distance between nanocompartments of up to $30 \mathrm{~nm}$ for mimicking the size of MCSs region between two organelles, ${ }^{12}$, 53 (ii) prevent membrane fusion to preserve the individual organelles, ${ }^{12}, 53$ and (iii) avoid aggregation. These requirements will select synthetic nano-compartments as ideal candidates with properties mimicking those of biocompartments (stable and flexible membrane, hollow 
spherical architecture, preserved integrity upon self-organization), such as organelles or cells. To fulfill the bio-related criteria results in a completely different approach compared with those used when other nano-objects (nanoparticles, quantum dots or nanorods) have been self-organized into networks. ${ }^{24,32}$ To achieve this goal, we selected polymersomes as nano-compartments that are generated by self-assembly of amphiphilic block copolymers, and which were functionalized to expose binding sites at their surface for single-strand DNA (ssDNA) attachment. Upon mixing of the complementary ssDNA-polymersomes, the hybridization serves to self-organize them into spatial supramolecular topologies yet unreported for synthetic polymersomes.

There are already advances in interconnecting liposomes or cell membranes via DNA hybridization. ${ }^{54-56}$ We selected polymersomes instead of liposomes as nano-compartments to be self-organized by DNA hybridization to take advantage of their mechanic stability, which will favor translational applications. However, the significant difference in the membrane thickness and thermodynamic properties of polymersomes compared with liposomes induces an increased degree of difficulty in the self-organization process of polymersomes by DNA hybridization, which prevents an extrapolation of the achievements already reported for interconnected liposomes. ${ }^{54-56}$ For example, the lateral diffusion in a polymer membrane is more than one order of magnitude lower than the one in a lipid membrane. ${ }^{57}$ For a successful insertion of biomolecules inside the synthetic membrane both the low lateral diffusion and the significant hydrophobic mismatch have to be overcome by carefully selection of the chemical nature of the copolymers. Moreover, to control the self-organization process we selected completely synthetic copolymers instead of copolymers that DNA serves as the hydrophilic block, ${ }^{58,59}$ which does not allow the modulation of the DNA surface distribution at the polymersomes surface, and might assemble without a compartment-like architecture. ${ }^{58,59}$ 
DNA provides high specific recognition between complementary ssDNA-polymersomes and the rigid structure of double-stranded DNA (dsDNA) is intended to control the distance between polymersomes up to $30 \mathrm{~nm}$, suitable for mimicking MCSs. In addition, the dsDNA bridge between polymersomes is intended to act as an isolation layer preventing membrane fusion. ${ }^{60} \mathrm{We}$ evaluated the influence of various molecular- (DNA surface density and size of polymersomes) and external factors (amount of polymersomes and hybridization temperature) on the selforganization process of ssDNA-polymersomes to control the size of the resulting assemblies and their topology. The difference between the flexibility of the polymersome membrane and the intrinsic rigidity of nanoparticles induces a completely different scenario of the self-organization process, resulting in clusters with properties mimicking biocompartments (flexible membrane, stable hollow-sphere architecture). On the other hand, the reduced flexibility of the polymersome membranes compared with lipid bilayers will prevent fusion, and mimic the natural organelle integrity as compartments.

In addition, the influence of molecular factors (surface density of ssDNA/polymersome, flexibility of the synthetic membrane and size of the polymersomes), on the specific conditions selected for development of our self-organized polymersomes prevents their aggregation, resulting in a hierarchically controlled assembly. Such polymersome clusters present the unique advantage over the reported networks of nano-objects to allow further development of reactions inside their cavity, by using the artificial organelle models already reported for single polymersomes. ${ }^{35}$ Polymersome clusters represent an essential step in development of interconnecting artificial organelles because they will topologically favor cascade reactions between different polymersomes and support a biomimetic strategy that is specific for cell signaling or interactions. In addition, our strategy based on synthetic nano-compartments instead 
of lipidic ones will serve for translational applications, which might be hindered by the intrinsic instability of the lipid bilayers. The straightforward control over the self-organization process by changing the DNA sequences exposed at the surface of polymersomes will serve for development of more complex and multifunctional architectures.

In order to obtain ssDNA-polymersomes, in situ modification of assembled polymersomes with ssDNA through strain-promoted azide-alkyne cycloaddition (SPAAC) was performed. This strategy avoids DNA pre-functionalization of the block copolymers, which would alter the ratio between hydrophilic and hydrophobic blocks and thereby disturb the self-assembly process. In order to generate polymersomes poly(2-methyloxazoline)-block-poly(dimethylsiloxane)-blockpoly(2-methyloxazoline $) \quad\left(\mathrm{PMOXA}_{7}-\mathrm{PDMS}_{42}-\mathrm{PMOX}_{7}\right) \quad$ triblock $\quad$ copolymer and poly(dimethylsiloxane)-block-poly(2-methyloxazoline) (PDMS $\left.75-\mathrm{PMOXA}_{37}-\mathrm{PEG}_{3}-\mathrm{N}_{3}\right)$ diblock copolymer were synthesized and mixed to self-assemble in dilute aqueous solution (Figure S1, $\mathrm{S} 2$ ). The terminal azide group of the $\mathrm{PDMS}_{75}-\mathrm{PMOXA}_{37}-\mathrm{PEG}_{3}-\mathrm{N}_{3}$ copolymer enables the linkage of the ssDNA via terminal dibenzocyclooctyne (DBCO) functionality. In addition, the extended PMOXA part serves both to favor the azide accessibility for the reaction with DBCOssDNA and as a spacer between the polymersome surface and the DNA to assure the accessibility of the linked ssDNA for hybridization (Figure 1a,b), in a similar manner as the spacer reported for DNA-functionalized nanoparticles. ${ }^{61}$

To maximize the number of azides exposed at the external surface of polymersomes, whilst avoiding to disturb the self-assembly process, $\mathrm{PMOXA}_{7}-\mathrm{PDMS}_{42}-\mathrm{PMOXA}_{7}$ was mixed with different amounts of $\mathrm{PDMS}_{75}-\mathrm{PMOXA}_{37}-\mathrm{PEG}_{3}-\mathrm{N}_{3}(0.00,0.25,1.00,5.00$ and $10.00 \mathrm{~mol} \%$, coded as $\mathrm{P} 0, \mathrm{P} 0.25, \mathrm{P} 1, \mathrm{P} 5$ and $\mathrm{P} 10$, respectively), and self-assembled by a film-rehydration method. ${ }^{36}$ Vesicular structures were observed in transmission electron microscopy (TEM) and 
cryo-TEM micrographs for initial molar contents of $\mathrm{PDMS}_{75}-\mathrm{PMOXA}_{37}-\mathrm{PEG}_{3}-\mathrm{N}_{3}$ below or equal to $5.00 \mathrm{~mol} \%$ (Figure S3a-d, g). They have a hydrodynamic diameter $\left(D_{\mathrm{H}}\right)$ of $180 \pm 60 \mathrm{~nm}$ (obtained by dynamic light scattering, DLS), independent of the molar fraction of PDMS ${ }_{75-}$ $\mathrm{PMOXA}_{37}-\mathrm{PEG}_{3}-\mathrm{N}_{3}$ (Table $\mathrm{S} 1$ ). These values are in agreement with the $D_{\mathrm{H}}$ values obtained by analysis of the TEM micrographs (the slight difference being inherent for TEM and DLS methods). The morphology transited toward worms and micelles for $\mathrm{PDMS}_{75}-\mathrm{PMOXA}_{37}-\mathrm{PEG}_{3}-$ $\mathrm{N}_{3}$ content of $10.00 \mathrm{~mol} \%$, whilst $\mathrm{PDMS}_{75}-\mathrm{PMOXA}_{37}-\mathrm{PEG}_{3}-\mathrm{N}_{3}$ formed rod-like micelles (Figure $\mathrm{S} 3 \mathrm{e}, \mathrm{f})$. We evaluated the molar ratio of $\mathrm{PDMS}_{75}-\mathrm{PMOXA}_{37}-\mathrm{PEG}_{3}-\mathrm{N}_{3}$ in the polymersome membrane by coupling DBCO-PEG4-Fluor 545 to the azide groups exposed at the polymersome surface through SPAAC. The brightness of DBCO-PEG4-Fluor 545-coupled to polymersomes was compared with that of the free DBCO-PEG4-Fluor 545. The average number of azide groups per polymersome for P0.25, P1 and P5 was determined as: $21 \pm 1,45 \pm 5$ to $121 \pm 7$, which corresponds to $0.2,0.3$ and 0.9 mol $\%$ of $\mathrm{PDMS}_{75}-\mathrm{PMOXA}_{37}-\mathrm{PEG}_{3}-\mathrm{N}_{3}$ present in the membrane (Table S2).

Subsequently, P0.25, P1 and P5 were post-functionalized through SPAAC with 22-mers of dibenzocyclooctyl-terminated ssDNA (ssDNAa) or the complementary strand (ssDNAb) (Figure 1b). A maximum yield of conversion was reached after 2 days, and no further significant increase was achieved by extending the reaction time (Figure S4). The reaction rate for the present system is lower than reported elsewhere ${ }^{62}$ for two reasons: (i) due to the click reaction being constrained to the polymersome surface and (ii) due to low content of PDMS 75 -PMOXA 37 $\mathrm{PEG}_{3}-\mathrm{N}_{3}$ in the polymersomes, desired to avoid disruption of the polymersome architecture and overpopulation with DNA. Both ssDNAa and ssDNAb were successfully bound to all P0.25 - P5 polymersomes, with coupling yields ranging from $27 \%$ to $75 \%$ of the initial azide-group 
amount (Table S1). The conjugation of ssDNAa to the polymersomes was proven by agarose gel electrophoresis where the appearance of a new band after cycloaddition reaction corresponds to the fraction of ssDNA bound to polymersomes (Figure S5). The variation of the coupling yields results from a combination of molecular factors, such as distribution of the surface density of the azide groups, their accessibility for SPAAC reaction, and the number of polymersomes present/solution volume. In addition, the increase of the content of $\mathrm{PDMS}_{75}-\mathrm{PMOXA}_{37}-\mathrm{PEG}_{3}-\mathrm{N}_{3}$ induces the appearance of a minor population of micelles by self-assembly instead of coassembly with $\mathrm{PMOXA}_{7}-\mathrm{PDMS}_{42}-\mathrm{PMOX}_{7}$. This hinders the expected increase of the number of azide groups exposed on the polymersome surface, resulting in a lower coupling yield for ssDNA on P5 (Table S1).

However, the increase of DNA density on polymersomes surface from P0.25-ssDNAa to P5ssDNAa is clearly indicated by the raise of the respective zeta-potential values from $-3.7 \pm 1.0$ $\mathrm{mV}$ to $-9.2 \pm 1.3 \mathrm{mV}$ (Table $\mathrm{S} 1$ ). No influence on the polymersome morphology or size was observed after DNA functionalization both by TEM and DLS (Figure 1c, Figure S6, Table S1).

In order to evaluate the average ssDNA number per polymersome and to determine the distribution of the DNA surface density $(\sigma)$, the hybridization of atto550-labelled complementary ssDNA (atto550-ssDNAb) with ssDNAa on the polymersomes was investigated by FCS (Figure $1 b, d)$. The significant increase of the diffusion time of the free atto550-ssDNAb $\left(\tau_{\mathrm{D}}=0.1 \mathrm{~ms}\right)$ to values of $4.3 \pm 0.2,4.7 \pm 0.2$ and $5.3 \pm 0.8 \mathrm{~ms}$ for atto550-ssDNAb hybridized to P0.25-ssDNAa, P1-ssDNAa and P5-ssDNAa, respectively, indicates successful hybridization of the complementary ssDNAb to the ssDNAa exposed at the surface of polymersomes. Similarly, atto647N-ssDNAa was hybridized to ssDNAb-functionalized polymersomes (Table S3). In both cases the hybridization of the complementary ssDNA to the ssDNA-polymersomes did not affect 
the polymersome architecture (Figure S7). No unspecific binding of atto550-ssDNAb to P5 polymersomes or of atto647N-ssDNAa to P5-ssDNAa was observed by FCS analysis 12 hours after mixing (Table S3). The average number of ssDNA per polymersome obtained by brightness measurements was calculated by dividing the CPM of fluorescently labeled ssDNA hybridized to polymersomes with the CPM corresponding to the free fluorescently labeled ssDNA (Table S3). The number of ssDNA per polymersome increased from $14 \pm 1$ for P0.25-ssDNAa to $93 \pm 2$ for P5-ssDNAa, and from $18 \pm 2$ for P0.25-ssDNAb to $127 \pm 9$ for P5-ssDNAb, respectively, depending on the amount of azide groups present on the polymersomes surface. The number of ssDNA/polymersome corresponds to an average $\sigma$ value from 0.1 to 1.2 strands per $1000 \mathrm{~nm}^{2}$ (Table S3). Note that the low values of the surface density of the ssDNA/polymersome were selected to avoid DNA repulsive interactions, and preserve the polymersome architecture yet still allowing hybridization. 

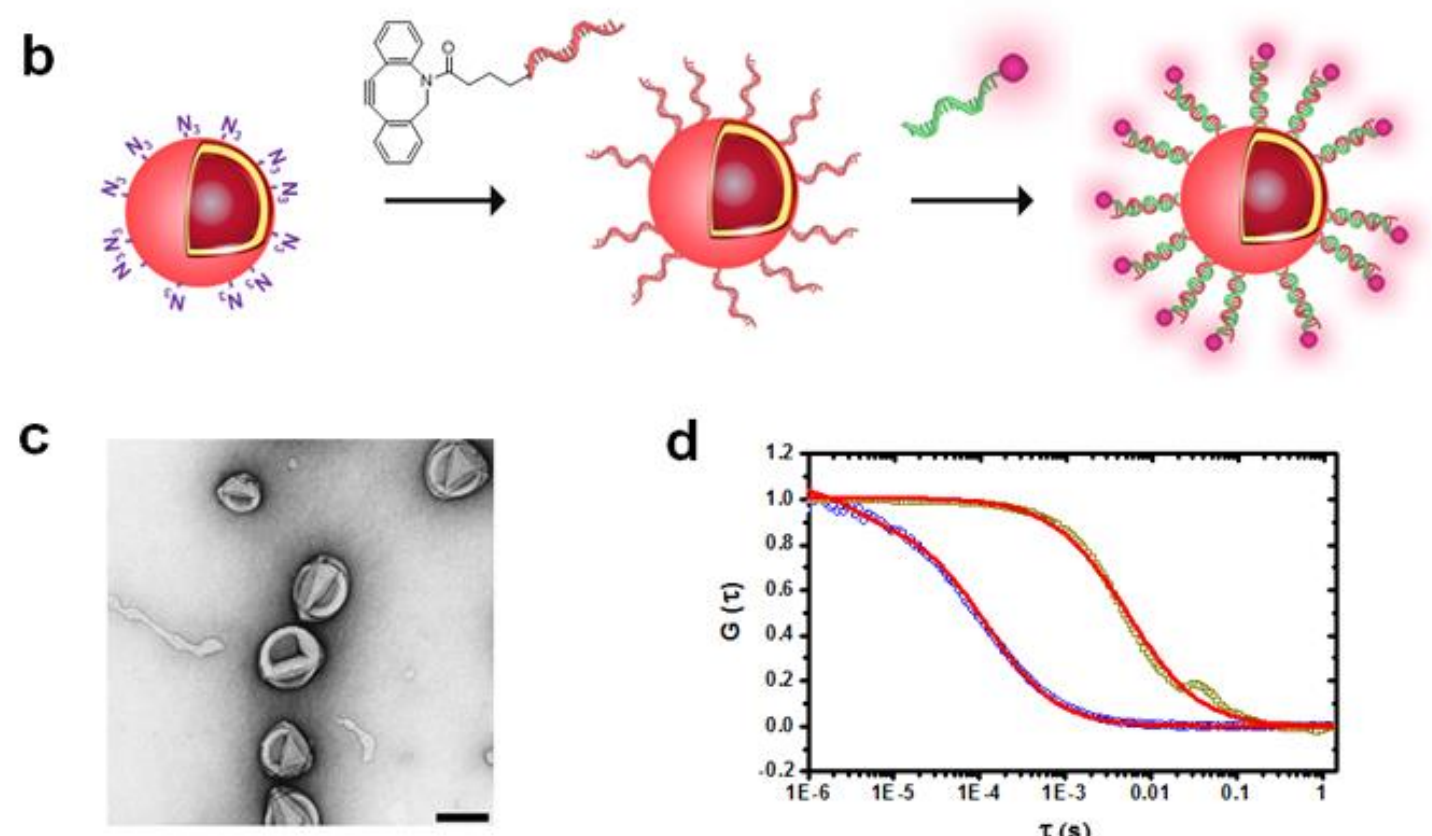

\section{5'-atto550-TAA CAG GAT TAG CAG AGC GAG G-3'}

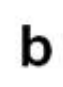

Figure 1. DNA-functionalized polymersomes. (a) Chemical structures of $\mathrm{PMOXA}_{7}-\mathrm{PDMS}_{42}-$ $\mathrm{PMOXA}_{7}$ and $\mathrm{PDMS}_{75}-\mathrm{PMOXA}_{37}-\mathrm{PEG}_{3}-\mathrm{N}_{3}$ and the sequences of ssDNAa and atto550-ssDNAb. (b) Schematic representation of polymersomes with azide groups on the surface, to which ssDNAa is bound, and further hybridized with atto550-ssDNAb. (c) TEM micrograph of P5ssDNAa, the scale bar is $200 \mathrm{~nm}$. (d) Normalized FCS autocorrelation curves of atto550ssDNAb (20 nM, blue) and atto550-ssDNAb hybridized to P5-ssDNAa (dark yellow) with their respective fits (red). 
In order to explore the DNA mediated self-organization of ssDNA-polymersomes, P0.25, P1 and P5 were mixed in equal volume fractions with the respective complementary polymersomes, hybridized at $37{ }^{\circ} \mathrm{C}$ and characterized by a combination of DLS and TEM (Figure 2). A rapid increase of $D_{\mathrm{H}}$ to a plateau was observed, indicating the self-organization of polymersomes in clusters by DNA hybridization for all ssDNA-polymersomes mixed with their complementary ssDNA-polymersomes (Figure 2b). Interestingly, polymersomes self-organized into submicrometer sized clusters (a major population of small clusters and a minor one of bigger clusters), and no aggregation was observed in time (Figure 2d), contrary to previous reported aggregates of DNA-liposomes ${ }^{51}$. An apparent $D_{\mathrm{H}}$ of $290 \pm 100 \mathrm{~nm}$ was measured for P5-ssDNAa - P5-ssDNAb clusters (P5-ab) at equilibrium by DLS $(n=5$, Figure S8c). The hybridization temperature of $37{ }^{\circ} \mathrm{C}$ was chosen to be well below the DNA's melting temperature of $67{ }^{\circ} \mathrm{C}$ (Figure S9) and to demonstrate the cluster formation under physiological conditions. Note that the relatively high distribution of the apparent values of the $D_{\mathrm{H}}$ is due to the intrinsic size distribution of polymersomes (Table S1). This appealing architecture of small clusters is exactly the desired one, when internalization of such clusters into cells is intended to develop translational applications.

The distribution of the number of polymersomes per cluster determined from a statistical analysis of TEM micrographs (Figure S10, S11, n = 200) corresponds to a binomial distribution. An average number of $2.2 \pm 1.5,2.0 \pm 1.3$ and $1.7 \pm 1.1$ polymersomes/cluster was calculated for P5-ab, P1-ab and P0.25-ab, respectively. The number of free polymersomes decreases with time; after 6 hours, an unbound fraction of $33 \pm 8 \%$ was present for P0.25, whilst for P1 and P5 this fraction was $21 \pm 11 \%$ and $11 \pm 7 \%$, respectively (Figure S11). 
In order to evaluate the influence of molecular- ( $\sigma$ value and size of polymersomes) and external- factors (the amount of polymersomes and temperature) on the self-organization process, the change in apparent $D_{\mathrm{H}}$ of clusters with respect to time was fitted by a doubleexponential function (Figure 2b,c, eq 1, Supporting Information). Two rate constants (a fast $k_{1}$ and a slow $k_{2}$ defined according to eq 2, Supporting Information) indicate the occurrence of two different stages during the organization process (Table S4). In the initial stage, polymersomes underwent a rapid self-organization with a short dwell time $\left(t_{1}=0.12 \mathrm{~s}, 0.16 \mathrm{~s}\right.$, and $0.21 \mathrm{~s}$ for P5$\mathrm{ab}, \mathrm{P} 1-\mathrm{ab}$ and P0.25-ab). The increase of the $\sigma$ value of polymersomes (from P0.25-ab to P5-ab) accelerated the initial self-organization step, as indicated by a higher $k_{1}$ value for P5-ab $\left(k_{1}=\right.$ $\left.8.33 \mathrm{~s}^{-1}\right)$, in comparison with the values corresponding to P0.25-ab and P1-ab clusters $\left(k_{1}=6.25\right.$ $\mathrm{s}^{-1}$ for $\mathrm{P} 1-\mathrm{ab}$, and $k_{1}=4.76 \mathrm{~s}^{-1}$ for $\left.\mathrm{P} 0.25-\mathrm{ab}\right)$. This behavior results from the increased probability of interaction between complementary ssDNA-polymersomes with an increased number of ssDNA/polymersome.

In order to investigate how the polymersome concentration affects the cluster formation, the polymersome solution was diluted 5 times prior to hybridization. The number of polymersomes/solution volume induced a slight reduction of $k_{1}$ (from $8.33 \mathrm{~s}^{-1}$ to $5.55 \mathrm{~s}^{-1}$ ), whilst $k_{2}$ was not affected. The decrease of the number of polymersomes in solution is expected to decrease the probability of their interaction, and consequently the $k_{1}$ value for the fast step of the self-organization process. A significant decrease of $k_{1}$ was observed when either the temperature for the formation of $\mathrm{P} 5-\mathrm{ab}$ was reduced from $37^{\circ} \mathrm{C}$ to $25^{\circ} \mathrm{C}$ or when the polymersome diameter was reduced from $180 \pm 60 \mathrm{~nm}$ to $110 \pm 30 \mathrm{~nm}$ (Table S4). The reduced hybridization temperature resulted in an expected decrease of $k_{1}$ (from $5.55 \mathrm{~s}^{-1}$ to $0.18 \mathrm{~s}^{-1}$ ) and ended with the same size of clusters as when formed at $37^{\circ} \mathrm{C}$ (Figure 2c, magenta vs cyan curves). The latter 
effect has as its main cause the difference in the number of ssDNA/polymersome when their size is reduced. As expected, the size of the polymersome clusters decreased when the concentrations of polymersomes, available for hybridization, was reduced five times (Figure 2c, grey and cyan curves).

The second step of the self-organization process is characterized by a plateau in $D_{\mathrm{H}}$ values with a low rate of cluster formation. $k_{2}$ values are significantly lower than $k_{1}$ values for all polymersomes with no obvious dependence on the $\sigma$ value, the size of polymersomes, the polymersome concentration or the temperature (Table S4). The plateau indicates a stabilization of the size of the clusters, and explains the lack of aggregation, which has been observed for DNA-liposomes ${ }^{63}$. This interesting stabilization of small clusters results from the specificity of conditions for the self-organization process based on relatively low number of ssDNA/polymersomes. Various other molecular factors, such as the concentration of complementary ssDNA-polymersomes in the mixture, and the distribution of the DNA surface density play a role in the formation of such small clusters and lack of aggregation. Both a reduced number of ssDNA-polymersomes and a fraction of polymersomes with low number or no DNA/polymersome decrease the probability of interaction between complementary ssDNApolymersomes, and therefore represent "dilution" factors limiting the formation of clusters in the second step of the self-organization process. 
a

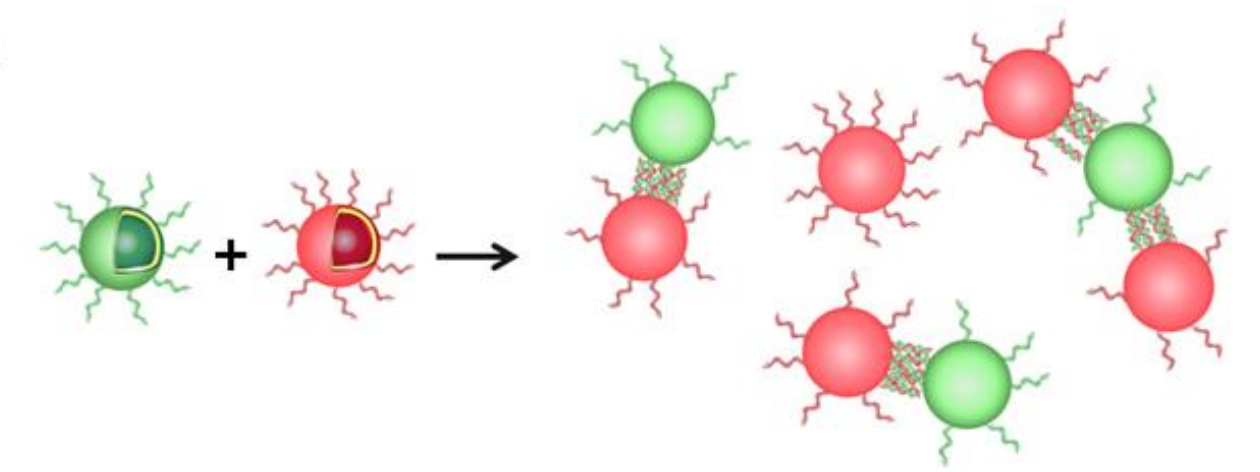

b

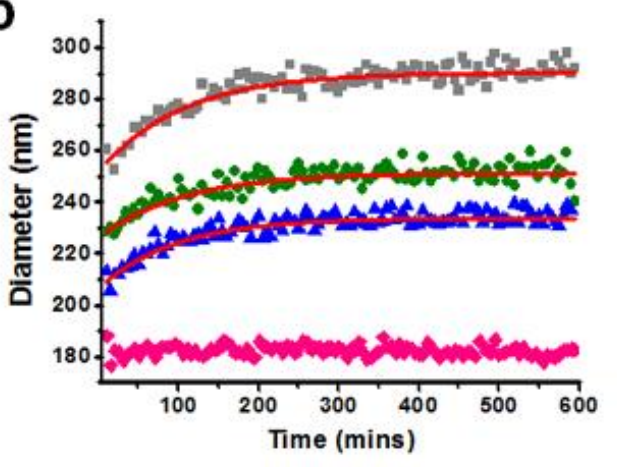

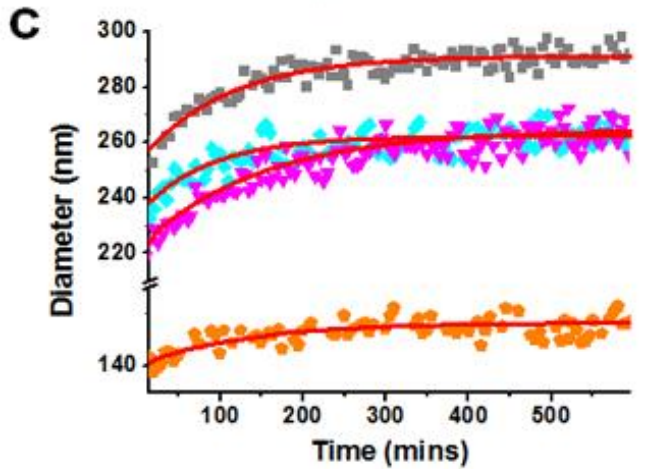
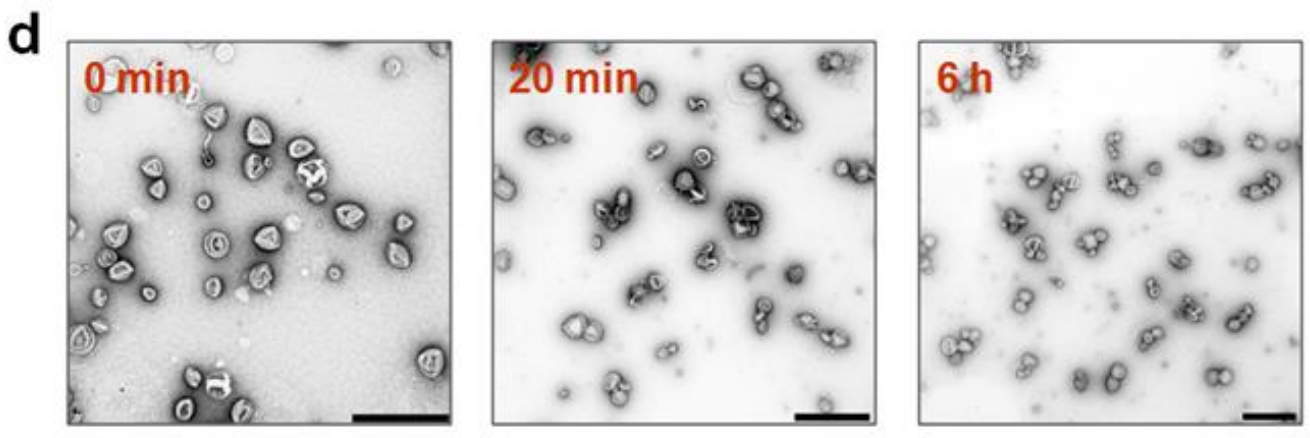

Figure 2. Self-assembly of complementary ssDNA-polymersomes into clusters. (a) Schematic representation of ssDNA-polymersome assembly by DNA hybridization; (b) Self-organization processes of P5-ab (gray), P1-ab (green), P0.25-ab (blue) and free ssDNAa-polymersomes (pink) by change of $D_{\mathrm{H}}$ as a function of the time at $37{ }^{\circ} \mathrm{C}$. (c) Illustration of the self-organization process of P5-ab at five times more diluted polymersome concentration (compared to the grey trace) at $37{ }^{\circ} \mathrm{C}$ (cyan), at $25^{\circ} \mathrm{C}$ (magenta) and with a size of $110 \pm 30 \mathrm{~nm}$ at $37{ }^{\circ} \mathrm{C}$ (orange) by reporting the change of $D_{\mathrm{H}}$ as a function of the time. The curves were fitted by a double- 
exponential function (red lines). (d) Self-organization of P5-ab at 0 min, after 20 min and after 6 $\mathrm{h}$, monitored by TEM. The scale bars are $1000 \mathrm{~nm}$.

In order to obtain more insight into the molecular factors favoring the self-organization process, the assembled clusters were analyzed by cryo-TEM (Figure S12). Unexpectedly, the growth of polymersome clusters induced a deformation of the polymeric membrane of interconnected ssDNA-polymersomes. The micrographs revealed the formation of an extended region of DNA bridges between interconnected polymersomes. This observation suggests that the initial binding of two complementary polymersomes is followed by a migration of DNA bearing polymer chains within the membrane. Such migration is supported by the flexibility of the PMOXAPDMS-PMOXA polymersome membrane, with high lateral diffusion coefficients for polymer chains inside the membrane. ${ }^{64}$ The concentration of DNA strands to form extended bridge domains between the interacting polymersomes represents a completely different phenomenon, not previously observed for other DNA-based networks/assemblies (e.g. nanoparticles ${ }^{65}$ ) where the position of the ssDNA is fixed by specific binding sites. In addition, such migration of the DNA strands is expected to induce an inhomogeneous distribution of the ssDNA/polymersome surface with a significant decrease of the number of DNA strands present at surface regions opposite to the binding region. This migration induced asymmetry in the DNA distribution on clustered polymersomes is one of the key factors hindering further growth of the clusters. It has been proposed in the case of nanoparticle assemblies that increasing elastic repulsive forces between linked nanoparticles and their oscillation towards different directions might induce instability of the assemblies. ${ }^{66,67}$ However, in case of the very flexible membrane of our polymersomes the described membrane deformation is not leading to polymersome rupture and does therefore not affect the stability of the formed clusters. However, the repulsive forces 
between the negatively charged phosphate groups in the DNA backbone are expected to play a role in determining the final size of the clusters. Together with the other molecular factors mentioned above it becomes evident that the process of DNA mediated polymersome cluster formation is rather complex. Further experiments are planned to understand and reveal the details of the self-organization process and the role of molecular factors in directing the clustering towards a specific topology.

In nature, it is fundamental to colocalize specific compartments (organelles) in a defined spatial organization to accomplish specific metabolic pathways and cell functions. In order to direct the self-organization of polymersomes we exploited two driving forces, DNA hybridization and steric hindrance created by different sized polymersomes. It has been demonstrated that steric hindrance is a key factor directing the assembly of particles into specific configuration. ${ }^{68}$ Two distinctly different ssDNA-polymersome populations, one with a diameter of $180 \pm 60 \mathrm{~nm}$ and a second one with a diameter of $110 \pm 30 \mathrm{~nm}$, were selected in order to create different steric hindrance (Figure 3a). For the visualization of the resulting clusters, atto488 and DY-633 dyes were encapsulated in P5-ssDNAb and P5-ssDNAa (P5-ssDNAbatto488, P5-ssDNAa-DY-633), respectively. The assembled clusters were immobilized on amino functionalized glass slides at $\mathrm{pH} 7.4$ by electrostatic interaction between the negatively charged DNA backbone and the positively charged amino-glass surface. The immobilization of the clusters did not lead to any observable polymersome rupture and allowed to record confocal laser scanning microscopy (CLSM) images to reveal the different cluster configurations by their respective fluorescence patterns. (Figure 3c,e). When complementary ssDNA-polymersomes with a diameter of $180 \pm 60 \mathrm{~nm}$ were mixed in an equal mass ratio, only clusters with a $D_{\mathrm{H}}$ of $290 \pm 100 \mathrm{~nm}$ and no large aggregates were observed by DLS after reaching the equilibrium 
(Figure S13c). Small chains of $2-4$ polymersomes $(2.2 \pm 1.5 ; \mathrm{n}=200)$ were observed by TEM and CLSM (Figure 3b,c). The relatively high polydispersity of the $D_{\mathrm{H}}$ of the assembled clusters can be explained by the dispersity of the polymersomes' $D_{\mathrm{H}}$ and the number of the assembled polymersomes. A completely different topology was observed by TEM and CLSM when nonequivalently sized polymersomes were hybridized: 2 - 6 small polymersomes $(3.8 \pm 1.9 ; \mathrm{n}=$ 230) hybridized onto the surface of a large polymersome, resembling a satellite like organization around a distinct central polymersome (Figure 3d,e, Figure S14). In addition, the electrostatic repulsive forces generated by DNA on the surrounding small polymersomes result in a large separation between individual satellites without any signs of uncontrolled aggregation (DLS: Figure S13e, TEM: Figure 3d). The distinct cluster topologies and the characteristics of the building blocks used to assemble them identifies steric hindrance, electrostatic repulsion and polymer chain migration as major driving forces behind the cluster architectures. The assembly of linear clusters is driven by polymer chain migration whereas the satellite configuration is governed by steric hindrance and electric repulsion between neighboring satellites. 
a

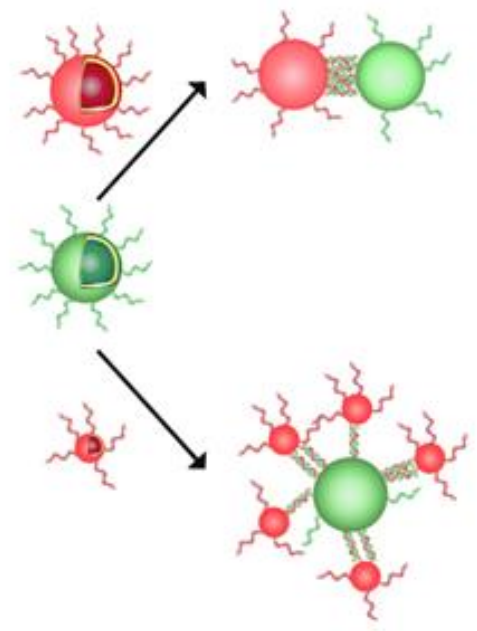

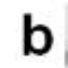
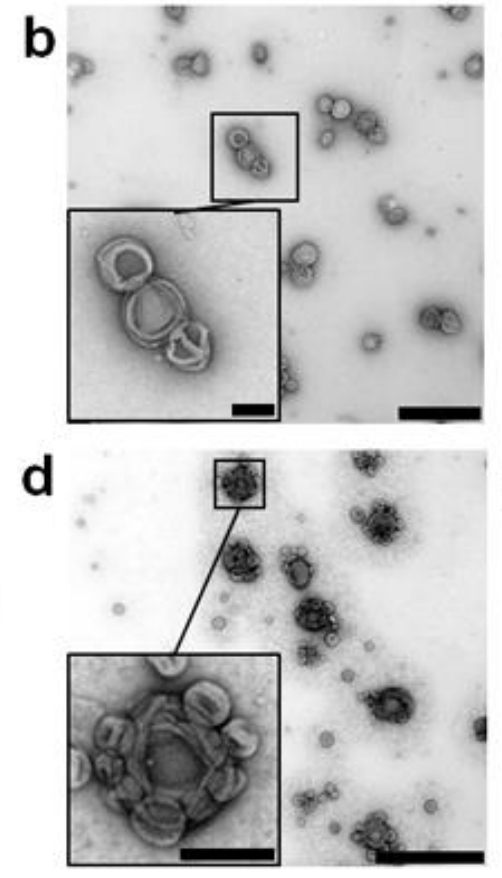

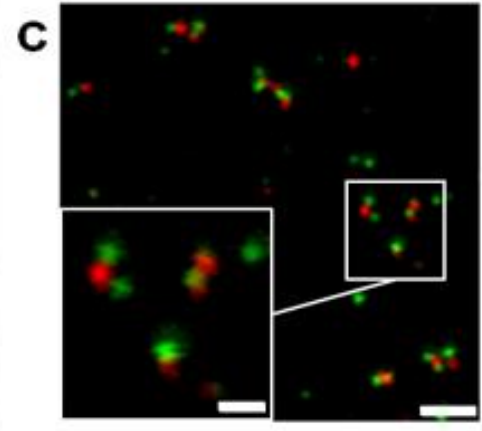

e

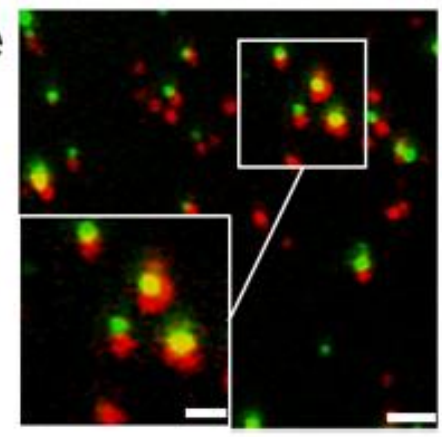

Figure 3. Self-organization of complementary ssDNA-polymersomes. (a) Schematic representation of distinct spatial topology resulting from mixing differently sized complementary ssDNA-polymersomes. TEM and CLSM micrographs of chain-like (b, c) and satellite-like polymersome clusters $(\mathrm{d}, \mathrm{e})$. The scale bar for TEM micrographs is $1000 \mathrm{~nm}$ and $200 \mathrm{~nm}$ in the inset, in CLSM micrographs it is $2000 \mathrm{~nm}$ and $1000 \mathrm{~nm}$ in the inset.

The spatial distance between ssDNA-polymersomes was manipulated by the length of ssDNA on the polymersomes exploiting the rigid nature of dsDNA ${ }^{69}$ (Figure 4). Complementary ssDNA with a length of either 22- or 44-mer were coupled to large P5 $(180 \pm 60 \mathrm{~nm})$, and subsequently hybridized. The distances between ssDNA-polymersomes sustained by 22-mer dsDNA and 44mer dsDNA were determined as $6.8 \pm 0.8 \mathrm{~nm}$ and $13.1 \pm 1.7 \mathrm{~nm}$ by the analysis of cryo-TEM images (Figure 4, n=132), in good agreement with the theoretical values of $7.48 \mathrm{~nm}$ and 14.96 $\mathrm{nm}$ assuming $3.4 \mathrm{~nm}$ per 10 base pairs, ${ }^{70}$ respectively. Detailed image analysis of the region of the bridges between connected polymersomes revealed the presence of dark thin bands, which 
can be associated with the dsDNA strands. ${ }^{71}$ Mean distance between them was estimated as: 5.83 $\pm 1.81 \mathrm{~nm}$, in agreement with the value of $5.29 \mathrm{~nm}$, which has been theoretically obtained based on a length of the DNA strand with 22-mer of $7.48 \mathrm{~nm}$, and the hypothesis of a homogeneous distribution of DNA (Supplementary Information). We obtained the number of dark bands corresponding to dsDNA strands as $15.7 \pm 2.2$ (four connected polymersomes were measured). The mean value of the radius of the contact area between two connected polymersomes was measured as $\mathrm{R}=37.6 \pm 3.35 \mathrm{~nm}$, which corresponds to a circular contact area of $4442 \pm 35.3$ $\mathrm{nm}^{2}$. The resulting density of DNA strands/contact area was obtained as 13.9 DNA strands/1000 $\mathrm{nm}^{2}$ (with the hypothesis of homogeneous distribution of the DNA strands), which is one order of magnitude higher than the average $\sigma$ value from 0.1 to 1.2 strands per $1000 \mathrm{~nm}^{2}$ (obtained for uncoupled ssDNA-polymersomes). This huge difference in the density of DNA strands before and after the formation of the bridges clearly indicates the migration of the DNA strands to support the polymersomes coupling. In order to determine the density of dsDNA in the bridge region we calculated the force responsible for the polymersomes deformation upon their coupling via DNA hybridization (in the limit of small deformations), and divided it by the force corresponding to the formation of hydrogen bonds in a dsDNA strand with 22-mer (Supplementary Information). We obtained a maximum density of DNA strands/contact area of 16.2 DNA strands/1000 $\mathrm{nm}^{2}$. Both the calculated value of the DNA density in the contact region based on cryo-TEM micrographs analysis, and that obtained from a simple model of elastic deformation of the polymersomes indicate a significant migration of the DNA strands to form the bridges between the connected polymersomes.

The deformation of the membrane upon cluster formation does not induce membrane rupture, and the polymersomes preserve their overall architecture inside the clusters with the 
encapsulated fluorescent dyes well separated in distinct cavities, as observed by a combination of cryo-TEM and CLSM (Figure 3, Figure 4). To determine whether the hybridization of ssDNApolymersomes induces membrane fusion between the respective polymersomes we mixed ssDNA-polymersomes containing a fluorescent dye in a self-quenching concentration with complementary ssDNA-polymersomes containing only PBS (Figure S15, Figure S16). When ssDNA-polymersomes containing self-quenched sulforhodamine B (SRB, $25 \mathrm{mM}$ ) were mixed with the complementary ones without dye, no increase of the fluorescence was observed after 5 days. Therefore, the clusters were stable for several days, with no leakage of the encapsulated content and no mixing of their content as it might result from their fusion. FRET analysis indicated the hybridization of DNA strands upon formation of clusters (Figure S17).

In comparison with polymersome aggregates linked by $\beta$-cyclodextrin and azobenzene, which undergo membrane fusion, ${ }^{72,73}$ the stability of DNA-polymersome clusters indicates that DNA acts not only as a linker to connect the polymersomes and to control the spatial distance, but also generates a protective layer, preventing efficiently the fusion of the polymeric membranes. This is an exciting property because it will allow encapsulation of different catalysts (enzymes, mimics) inside each type of polymersome to perform reactions either inside the polymersome or between different polymersomes from the same cluster (appropriately permeabilized to allow exchange of molecules) to gain multifunctionality in a controlled manner.
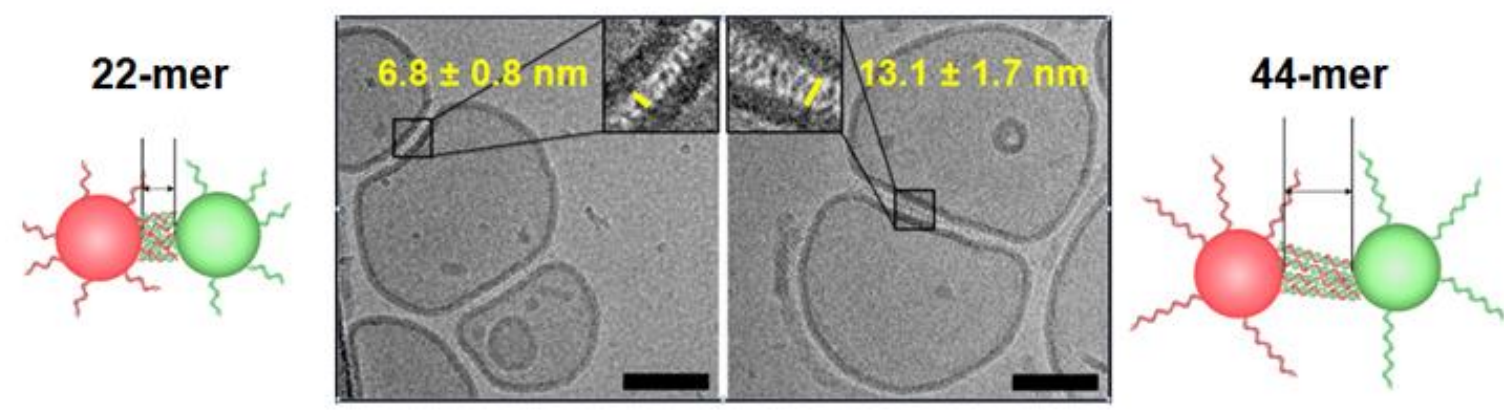
Figure 4. Schematic representation and cryo-TEM micrographs of DNA-polymersome clusters connected by 22-mer (left) and 44-mer (right) dsDNA, resembling the neuronal gap. The scale bars are $100 \mathrm{~nm}$.

Self-organization of nano-objects is a key tool to generate complex networks with novel topology and properties. We were interested to go one step further in using this process by adding new properties in a biomimetic approach based on connected organelles inside cells, known as essential super-structures involved in various metabolic processes. Our strategy consists in self-organizing artificial nano-compartments, specifically polymersomes composed of a completely synthetic membrane, in a controlled manner to mimic the organization of organelles with MCSs, and generate clusters with a topology according to design. By selecting DNA as the linkage molecule to drive the self-organization between polymersomes, the clusters generated by self-organization of complementary ssDNA-polymersomes exhibit features for mimicking connected organelles, such as high stability, no membrane fusion, no aggregation, and control over the distance between polymersomes due to the rigid feature of dsDNA. Interestingly, extended DNA bridges that result from the migration of the DNA strands inside the thick flexible polymersome membrane (about $12 \mathrm{~nm}$ thick) were revealed in the gap between connected polymersomes by cryo-TEM micrographs. They generate an asymmetry in the DNA distribution at the surface of polymersomes, and represent an important factor to stabilize and control the architecture and size of clusters, which has not yet been exploited for other synthetic nanoobjects networks. Interfacing polymersomes by reorganization of DNA strands to generate extended bridges is expected to allow further development of complex architectures by the play between compact and incompact DNA distribution together with the length and surface density of the DNA strands. 
In addition, by using artificial organelles (resulting from encapsulation/co-encapsulation of active compounds inside the clustered polymersomes) instead of empty polymersomes makes them ideal candidates for further development of in situ cascade reactions between different ones that cannot be achieved within other networks/clusters of nano-objects, such as nanoparticles, micelles or nanorods. This is a unique advantage for the development of novel materials exhibiting biomimetic features inside their hierarchical organization and a controlled architecture based on the straightforward change of molecular factors affecting the organization process (size of polymersome, surface density of DNA/polymersome, length of DNA strands, concentration). For example, these small ssDNA-polymersome clusters can be further optimized to provide by their architecture a highly promising platform for further cell mimicking such as emulation of the synaptic gap, signal transduction and $\mathrm{Ca}^{2+}$ storage considering the negative nature of DNA. In addition, our strategy provides control over the cluster formation by changing the DNA sequence in terms of size and specificity, in order to gain more complex architectures.

Spatial organization and arrangement of such DNA-polymersomes with different functions in a defined order is of essential significance both for mimicking the integration of organelles in living cells, and for further development of translational applications, required in domains such as medicine, catalysis and, technology.

\section{ASSOCIATED CONTENT}

\section{Supporting Information}

Material and Methods and supplementary figures and tables. This material is available free of charge via the Internet at http://pubs.acs.org. 


\section{AUTHOR INFORMATION}

\section{Corresponding Author}

*E-mail: cornelia.palivan@unibas.ch (C.G.P.).

\section{Author Contributions}

J.L. contributed to the clusters formation and characterization, and the writing of the manuscript; V.P. contributed to the writing the manuscript; S.L. contributed to triblock copolymer synthesis and characterization, and the writing of the manuscript; J.L., V.P., S.L. contributed to the preparation of graphics; D.W. contributed to functionalized copolymer synthesis and DNA binding to polymersomes; M.C. contributed to Cryo-TEM experiments; W.M. contributed to synthesis of copolymers and writing the manuscript, and C.G.P. contributed to the self-organization concept and writing of the manuscript.

\section{Notes}

The authors declare no competing financial interests.

\section{ACKNOWLEDGMENT}

We gratefully acknowledge the financial support provided by the Swiss National Science Foundation, and the National Centre of Competence in Research Molecular Systems Engineering. J.L. thanks Dr. G. Gunkel Grabole (University of Basel) for the preparation of the amino glass slides for CLSM, Dr. F. Itel and Dr. M. Spulber (University of Basel) for fruitful discussions, and G. Persy (University of Basel) for TEM measurements. C.G.P. thanks Dr. H. Palivan for modeling the elastic deformation of connected polymersomes. M.C. thanks to Prof. H. Stahlberg (C-Cina, Biozentrum, University of Basel) for his support, and the University of 
Basel SystemX.ch RTD CINA for generous financial support. Dr. B.A. Goodman is acknowledged for reading the manuscript.

\section{REFERENCES}

1. Karsenti, E. Nat. Rev. Mol. Cell Biol. 2008, 9, 255-262.

2. Sütterlin, C.; Colanzi, A. J. Cell Biol. 2010, 188, 621-628.

3. Manford, A. G.; Stefan, C. J.; Yuan, H. L.; MacGurn, J. A.; Emr, S. D. Dev. Cell 2012, 23, 1129-1140.

4. Stefan, C. J.; Manford, A. G.; Emr, S. D. Curr. Opin. Cell Bio. 2013, 25, 434-442.

5. Annunziata, I.; d'Azzo, A. Cells 2013, 2, 574-590.

6. Burgoyne, T.; Patel, S.; Eden, E. R. Biochim. Biophys. Acta 2015, 1853, 2012-2017.

7. Daniele, T.; Schiaffino, M. V. Commun. Integr. Biol. 2014, 7, e29587.

8. Helle, S. C. J.; Kanfer, G.; Kolar, K.; Lang, A.; Michel, A. H.; Kornmann, B. Biochim. Biophys. Acta 2013, 1833, 2526-2541.

9. Rowland, A. A.; Voeltz, G. K. Nat. Rev. Mol. Cell Biol. 2012, 13, 607-625.

10. Raiborg, C.; Wenzel, E. M.; Pedersen, N. M.; Olsvik, H.; Schink, K. O.; Schultz, S. W.; Vietri, M.; Nisi, V.; Bucci, C.; Brech, A.; Johansen, T.; Stenmark, H. Nature 2015, 520, 234238.

11. Chung, J.; Torta, F.; Masai, K.; Lucast, L.; Czapla, H.; Tanner, L. B.; Narayanaswamy, P.; Wenk, M. R.; Nakatsu, F.; De Camilli, P. Science 2015, 349, 428-432. 
12. Prinz, W. A. J. Cell Bio. 2014, 205, 759-769.

13. Nie, Z.; Petukhova, A.; Kumacheva, E. Nat. Nano. 2010, 5, 15-25.

14. Talapin, D. V.; Lee, J.-S.; Kovalenko, M. V.; Shevchenko, E. V. Chem. Rev. 2010, 110, $389-458$.

15. Kuzyk, A.; Schreiber, R.; Fan, Z.; Pardatscher, G.; Roller, E.-M.; Hogele, A.; Simmel, F. C.; Govorov, A. O.; Liedl, T. Nature 2012, 483, 311-314.

16. Young, K. L.; Ross, M. B.; Blaber, M. G.; Rycenga, M.; Jones, M. R.; Zhang, C.; Senesi, A. J.; Lee, B.; Schatz, G. C.; Mirkin, C. A. Adv. Mater. 2014, 26, 653-659.

17. Majetich, S. A.; Wen, T.; Booth, R. A. ACS Nano 2011, 5, 6081-6084.

18. Sun, S.; Murray, C. B.; Weller, D.; Folks, L.; Moser, A. Science 2000, 287, 1989-1992.

19. Hermanson, K. D.; Lumsdon, S. O.; Williams, J. P.; Kaler, E. W.; Velev, O. D. Science 2001, 294, 1082-1086.

20. Schmid, G.; Simon, U. Chem. Commun. 2005, 697-710.

21. Liu, W.; Tagawa, M.; Xin, H. L.; Wang, T.; Emamy, H.; Li, H.; Yager, K. G.; Starr, F. W.; Tkachenko, A. V.; Gang, O. Science 2016, 351, 582-586.

22. Cheng, W.; Park, N.; Walter, M. T.; Hartman, M. R.; Luo, D. Nat. Nanotechnol. 2008, 3, $682-690$.

23. Kim, Y.; Macfarlane, R. J.; Jones, M. R.; Mirkin, C. A. Science 2016, 351, 579-582. 
24. Zhang, C.; Macfarlane, R. J.; Young, K. L.; Choi, C. H. J.; Hao, L.; Auyeung, E.; Liu, G.; Zhou, X.; Mirkin, C. A. Nat. Mater. 2013, 12, 741-746.

25. Li, Y.; Liu, Z.; Yu, G.; Jiang, W.; Mao, C. J. Am. Chem. Soc. 2015, 137, 4320-4323.

26. Wang, Y.; Wang, Y.; Breed, D. R.; Manoharan, V. N.; Feng, L.; Hollingsworth, A. D.; Weck, M.; Pine, D. J. Nature 2012, 491, 51-55.

27. Xu, X.; Rosi, N. L.; Wang, Y.; Huo, F.; Mirkin, C. A. J. Am. Chem. Soc. 2006, 128, 9286-9287.

28. Xing, H.; Wang, Z.; Xu, Z.; Wong, N. Y.; Xiang, Y.; Liu, G. L.; Lu, Y. ACS Nano 2012, $6,802-809$.

29. Tian, Y.; Wang, T.; Liu, W.; Xin, H. L.; Li, H.; Ke, Y.; Shih, W. M.; Gang, O. Nat. Nanotechnol. 2015, 10, 637-644.

30. Zhang, T.; Neumann, A.; Lindlau, J.; Wu, Y.; Pramanik, G.; Naydenov, B.; Jelezko, F.; Schüder, F.; Huber, S.; Huber, M.; Stehr, F.; Högele, A.; Weil, T.; Liedl, T. J. Am. Chem. Soc. 2015, 137, 9776-9779.

31. Aldaye, F. A.; Palmer, A. L.; Sleiman, H. F. Science 2008, 321, 1795-1799.

32. Zhang, Y.; Lu, F.; Yager, K. G.; van der Lelie, D.; Gang, O. Nat. Nanotechnol. 2013, 8, $865-872$.

33. Chou, L. Y. T.; Zagorovsky, K.; Chan, W. C. W. Nat. Nanotechnol. 2014, 9, 148-155.

34. Soenen, S. J.; Parak, W. J.; Rejman, J.; Manshian, B. Chem. Rev. 2015, 115, 2109-2135. 
35. Palivan, C. G.; Goers, R.; Najer, A.; Zhang, X.; Car, A.; Meier, W. Chem. Soc. Rev. 2016, $45,377-411$.

36. Tanner, P.; Balasubramanian, V.; Palivan, C. G. Nano Lett. 2013, 13, 2875-2883.

37. Allen, T. M.; Cullis, P. R. Adv. Drug Deliv. Rev. 2013, 65, 36-48.

38. Martino, C.; Kim, S.-H.; Horsfall, L.; Abbaspourrad, A.; Rosser, S. J.; Cooper, J.; Weitz, D. A. Angew. Chem. Int. Ed. 2012, 51, 6416-6420.

39. Schoonen, L.; van Hest, J. C. M. Adv. Mater. 2016, 28, 1109-1128.

40. Stengel, G.; Zahn, R.; Höök, F. J. Am. Chem. Soc. 2007, 129, 9584-9585.

41. Versluis, F.; Voskuhl, J.; van Kolck, B.; Zope, H.; Bremmer, M.; Albregtse, T.; Kros, A. J. Am. Chem. Soc. 2013, 135, 8057-8062.

42. Chan, Y.-H. M.; van Lengerich, B.; Boxer, S. G. Proc. Nati. Acad. Sci. USA 2009, 106, 979-984.

43. Dewey, D. C.; Strulson, C. A.; Cacace, D. N.; Bevilacqua, P. C.; Keating, C. D. Nat. Commun. 2014, 5.

44. Wang, Z.; van Oers, M. C. M.; Rutjes, F. P. J. T.; van Hest, J. C. M. Angew. Chem. Int. Ed. 2012, 51, 10746-10750.

45. Thompson, K. L.; Chambon, P.; Verber, R.; Armes, S. P. J. Am. Chem. Soc. 2012, 134, 12450-12453.

46. Elani, Y.; Law, R. V.; Ces, O. Nat. Commun. 2014, 5. 
47. Roling, O.; Wendeln, C.; Kauscher, U.; Seelheim, P.; Galla, H.-J.; Ravoo, B. J. Langmuir 2013, 29, 10174-10182.

48. Dave, N.; Liu, J. ACS Nano 2011, 5, 1304-1312.

49. Jakobsen, U.; Simonsen, A. C.; Vogel, S. J. Am. Chem. Soc. 2008, 130, 10462-10463.

50. Serien, D.; Grimm, C.; Liebscher, J.; Herrmann, A.; Arbuzova, A. New J. Chem. 2014, $38,5181-5185$.

51. Beales, P. A.; Vanderlick, T. K. J. Phys. Chem. A 2007, 111, 12372-12380.

52. Loew, M.; Kang, J.; Dähne, L.; Hendus-Altenburger, R.; Kaczmarek, O.; Liebscher, J.; Huster, D.; Ludwig, K.; Böttcher, C.; Herrmann, A.; Arbuzova, A. Small 2009, 5, 320-323.

53. Elbaz, Y.; Schuldiner, M. Trends biochem. Sci. 2011, 36, 616-623.

54. Parolini, L.; Kotar, J.; Di Michele, L.; Mognetti, B. M. ACS Nano 2016, 10, 2392-2398.

55. Selden, N. S.; Todhunter, M. E.; Jee, N. Y.; Liu, J. S.; Broaders, K. E.; Gartner, Z. J. J. Am. Chem. Soc. 2012, 134, 765-768.

56. Weber, R. J.; Liang, S. I.; Selden, N. S.; Desai, T. A.; Gartner, Z. J. Biomacromolecules 2014, 15, 4621-4626.

57. Itel, F.; Chami, M.; Najer, A.; Lörcher, S.; Wu, D.; Dinu, I. A.; Meier, W. Macromolecules 2014, 47, 7588-7596.

58. Fujita, M.; Hiramine, H.; Pan, P.; Hikima, T.; Maeda, M. Langmuir 2016, 32, 1148-1154. 
59. Kamps, A. C.; Cativo, M. H. M.; Chen, X.-J.; Park, S.-J. Macromolecules 2014, 47, $3720-3726$.

60. Martens, S.; McMahon, H. T. Nat. Rev. Mol. Cell Biol. 2008, 9, 543-556.

61. Hurst, S. J.; Lytton-Jean, A. K. R.; Mirkin, C. A. Anal. Chem. 2006, 78, 8313-8318.

62. Saito, F.; Noda, H.; Bode, J. W. ACS Chem. Bio. 2015, 10, 1026-1033.

63. Beales, P. A.; Vanderlick, T. K. J. Phys. Chem. A 2007, 111, 12372-12380.

64. Itel, F.; Chami, M.; Najer, A.; Loercher, S.; Wu, D.; Dinu, I. A.; Meier, W. Macromolecules 2014, 47, 7588-7596.

65. Mucic, R. C.; Storhoff, J. J.; Mirkin, C. A.; Letsinger, R. L. J. Am. Chem. Soc. 1998, 120, 12674-12675.

66. Seth, J. R.; Cloitre, M.; Bonnecaze, R. T. J. Rheol. 2006, 50, 353-376.

67. Campbell, C. S. Granul. Matter 2003, 5, 129-134.

68. Zerrouki, D.; Baudry, J.; Pine, D.; Chaikin, P.; Bibette, J. Nature 2008, 455, 380-382.

69. Goodman, R. P.; Schaap, I. A. T.; Tardin, C. F.; Erben, C. M.; Berry, R. M.; Schmidt, C. F.; Turberfield, A. J. Science 2005, 310, 1661-1665.

70. Bao, G.; Suresh, S. Nat. Mater. 2003, 2, 715-725.

71. Tahara, Y.; Fujiyoshi, Y. Micron 1994, 25, 141-149.

72. Jin, H.; Zheng, Y.; Liu, Y.; Cheng, H.; Zhou, Y.; Yan, D. Angew. Chem. Int. Ed. 2011, 50, 10352-10356. 
73. Smart, T. P.; Fernyhough, C.; Ryan, A. J.; Battaglia, G. Macromol. Rapid Commun. 2008, 29, 1855-1860.

Table of Contents Graphic

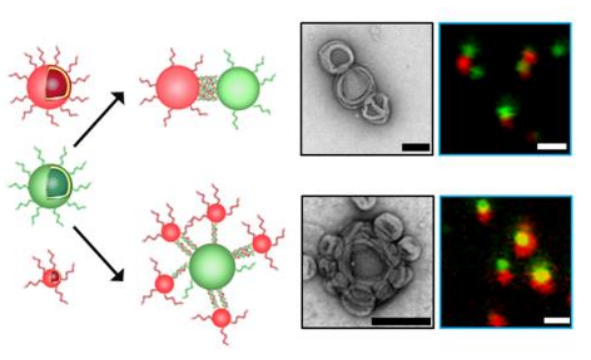

